\title{
Paul Ricoeur, pensador del testimonio histórico
}

\author{
(Paul Ricoeur: Thinker \\ of historical Testimony)
}

\author{
Esteban LYTHGOE
}

Recibido: 25 de noviembre de 2011

Aceptado: 13 de diciembre de 2011

\section{Resumen}

Este artículo propone una lectura ética de La memoria, la historia, el olvido. Se recurre al testimonio del Holocausto, en tanto acontecimiento injustificable, como instancia de reflexión ética originada en la historia. En estos términos, la respuesta de Ricoeur al Debate de los Historiadores adquiere una importancia capital. Nuestra hipótesis es que esta respuesta es el producto de una larga reflexión sobre las consideraciones de Jean Nabert con respecto a la relación entre la dimensión fáctica y la ética.

Palabras clave: ética, historia, injustificable, Holocausto, atestación.

\begin{abstract}
This paper proposes an ethical interpretation of Memory, History and Oblivion. Testimony of the Holocaust, as an unjustifiable event, is the historical point of departure of the ethical reflection. In these terms, Ricoeur's position in relation to the Historikerstreit acquires a great value. Our hypotheses is that this answer is the product of a long reflection on Jean Nabert's considerations on the relation of the factical and ethical dimensions.
\end{abstract}

Keywords: ethics, history, unjustifiable, Holocaust, attestation. 
Pero vemos de golpe la enormidad de la paradoja que la filosofía del testimonio suscita: “¿Tenemos el derecho, pregunta Nabert en el Ensayo sobre el mal (148) a investir de un carácter absoluto un momento de la historia?" ¿Cómo, en efecto, unir la interioridad de la afirmación originaria y la exterioridad de actos y de existencias que, se dice, testifican por lo absoluto? Es esta paradoja que la hermenéutica del testimonio trabaja para resolver. (Ricoeur 1972, p. 37).

Paul Ricoeur ha dedicado al menos dos artículos al problema del testimonio: "Hermenéutica del testimonio" y "Emmanuel Levinas, pensador del testimonio". Hay también algunas secciones de Sí mismo como otro referidas a este tema. Todos estos escritos se han constituido en el fundamento de una gran cantidad de artículos dedicados a destacar el componente ético del testimonio. Un caso es el de M. F. Begué quien, basándose en las implicaciones propias del acto de testificar, establece que el testimonio debe ser encuadrado dentro de un horizonte más amplio que el meramente epistémico. Su análisis la lleva a concluir que, “...entre un testigo fiel y verídico y un narrador escrupuloso hay diferencia. El testigo fiel no se limita a relatar 'tal cosa' sino que también rinde homenaje a algo, a una verdad que lo supera, cuyo compromiso le lleva más allá de la fría repetición de un relato.” (Begué 2006, p. 90).

En La memoria, la historia, el olvido el filósofo vuelve sobre la cuestión del testimonio. Sin embargo, a primera vista, su tratamiento resulta radicalmente diferente al de las obras arriba mencionadas. Este libro está dedicado fundamentalmente a la filosofía teórica: comienza con una fenomenología de la memoria, continúa con una epistemología de la historia y ambas quedan articuladas por medio de una ontología de la condición histórica. El testimonio, por su parte, es desarrollado en la sección dedicada a la epistemología de la historia. Este hecho por sí mismo permitiría justificar una lectura epistemologizante del testimonio, más afín a autores como Hume o Reid, antes que una lectura de corte ético ${ }^{1}$. Este tipo de interpretación no se vería afectada por el componente ético presente en la atestación ${ }^{2}$ pues, en definitiva, este atributo, ligado a la oposición fiabilidad - sospecha, es, al fin y al cabo, el tipo de creencia propio del testimonio, que, como modo aléthico, se opone al de la verdad como correspondencia 3 .

\footnotetext{
1 Cf. Lythgoe (2011).

2 Cf. Ricoeur (1996), p. XXXVI: "El parentesco entre atestación y testimonio se comprueba aquí: no hay 'verdadero' testigo sin 'falso' testigo. Pero no queda más recurso contra el falso testimonio que otro testimonio más creíble y no hay más recurso contra la sospecha que una atestación más fiable." ${ }^{3}$ Cf. Ricoeur (1996), p. XXXIV.
} 
El objetivo del siguiente artículo consiste en relativizar esta lectura epistemologizante de La memoria, la historia, el olvido. Esto significa que sostenemos que su temática principal no es aquella a la que se le ha dedicado mayor extensión, es decir, el problema de la relación entre la memoria y la historia, sino una a la que apenas se le han dedicado pocos párrafos separados entre sí y que, por lo tanto, es preciso reunirlos y articularlos. Se trata de la apertura de la dimensión ética a partir de la historia. Considero que este acercamiento a la obra no es arbitrario, y la primera pauta de ello nos la proporciona la importancia que en su desarrollo tiene el Holocausto,en tanto paradigma del acontecimiento injustificable. Desde esta perspectiva, la respuesta de Ricoeur al Debate de los Historiadores adquiere una relevancia mucho mayor que la que se le suele conceder. Nuestra hipótesis es que ella no es meramente contextual, sino el producto de una larga reflexión sobre las consideraciones hechas por Jean Nabert acerca de la relación entre la dimensión fáctica de la historia y la ética.

La tematización histórica del Holocausto ha generado gran cantidad de dificultades a esta disciplina. A la hora de llevar a cabo sus investigaciones, los historiadores suelen apoyarse en una base documental contrastable a la que tratarán de encontrarle una explicación o, de lo contrario, comprenderla. Pero, una narración histórica que se limite a estos dos puntos dejaría de lado lo aberrante e injustificable que resultó este acontecimiento, cuando sean justamente estos atributos los que lo definan de manera más cabal. El Holocausto posee un excedente de sentido, ligado a cuestiones éticas, que se le escapan a estos números. Jeffrey Alexander ha señalado con precisión esta tensión entre la dimensión meramente fáctica de la historia y la narración histórica del siguiente modo: "volverse mal es una cuestión, primero y principal, de representación. Dependiendo de la naturaleza de la representación, un acontecimiento traumático puede ser visto como ontológicamente maligno, o su maldad, puede ser concebida como contingente y relativa, como algo que puede ser mejorado y superado. Esta distinción es teórica, pero también práctica. De hecho, las decisiones sobre el carácter ontológico versus el contingente del Holocausto fueron de importancia fundamental en su cambio de representación." (Alexander 2004, p. 202).

Esta tensión entre la dimensión ética y la fáctica subyace en lo que se dio en llamar el Debate de los Historiadores y que tuvo lugar entre los años 1986 y 1988. En él, autores como Ernst Nolte y Jürgen Habermas discutieron en torno a la singularidad del Holocausto. El punto de conflicto podría resumirse con los siguientes términos: toda explicación consiste en subsumir un acontecimiento en un principio 
general aplicable a varias situaciones. La aplicación de este principio supone la posibilidad de comparar el Holocausto con otros acontecimientos, retirándole, consecuentemente, el calificativo de singular. El otro motivo utilizado para rechazar cualquier posible explicación de este acontecimiento es que proporcionaría una suerte de condonación a los perpetradores. Como en su momento afirmó Primo Levi, "Quizá no se pueda comprender todo lo que sucedió, o no se deba comprender, porque comprender casi es justificar." (Levi 2006, p. 240).

Ricoeur toma distancia de los términos en que se había planteado este debate, por considerarlo falazmente fundado. En su opinión, se recurría de manera indiscriminada a los múltiples sentidos del término singularidad y, gracias a esta vaguedad, se solapaban diferentes niveles discursivos. Por este motivo, la aclaración de los distintos sentidos utilizados acaba disolviendo gran parte de este debate.

En primer lugar, se señala la necesidad de distinguir la singularidad histórica de la moral: "la singularidad histórica no es la singularidad moral que hemos identificado más arriba como la inhumanidad extrema; esta singularidad por el exceso en cuanto al mal, que Nabert denomina lo injustificable y Friedlander lo inaceptable, no es ciertamente separable de rasgos históricos identificables; pero compete al juicio moral sacado, de alguna forma, de quicio." (Ricoeur 2000, p. 432).

Por su parte, el filósofo distingue dos sentidos diferentes de la singularidad histórica. El primero consiste en la no repetitividad. En un sentido trivial, toda serie causal contingente es singular. El solapamiento de este uso histórico del concepto con el de singularidad moral se realiza a través de la imputación de la acción al agente responsable. El uso de este sentido de singularidad ha dado lugar a la discusión entre los intencionalistas, que destacan la importancia de las decisiones tomadas en pos de la Solución Final, y los funcionalistas, para quienes los individuos son meros engranajes dentro la maquinaria de las instituciones sociales. La escuela intencionalista, con fuertes afinidades con la lectura judicial del Holocausto, sostiene la necesidad de imputar la realización de las acciones genocidas a agentes individualizados, asignándoles a ellos la responsabilidad moral de los actos. Los funcionalistas, por su parte, tienen una lectura más sociológica de este acontecimiento, en el sentido en que su atención está dirigida al funcionamiento de fuerzas anónimas, como lo son las diversas instituciones o el comportamiento social. En la medida en que esta escuela deja de lado la imputación del acto a un agente individualizado, sus explicaciones acaban siendo tomadas como exculpatorias. En sus consideraciones acerca del perdón, Ricoeur pone de manifiesto la existencia un abismo entre el acto y su agente. Lo que allí se sostiene es que ambas instancias sólo son articulables entre sí a través de una operación de segundo grado consistente en ligar y desligar el acto de su agente 4 . Esto significa que en ambos casos nos encontramos

4 Cf. Ricoeur (2000) pp. 595 y ss. La necesidad de tener presente la distinción entre acto y agente no sólo se aplicará a nivel de la imputabilidad tratada en esta sección, sino en el análisis de la memoria colectiva (155) y el perdón (637). 
frente una confusión categorial. No corresponde a la labor del historiador responsabilizar moralmente a un actor individualizado, así como tampoco exculparlo.

El segundo sentido de singularidad es el de incomparabilidad, que es otra manera de denominar a la unicidad. Según el filósofo francés, desde el punto de vista epistemológico, no tiene sentido hablar de una incomparabilidad, puesto que toda designación presupone una comparación previa que permita establecer similitudes y diferencias. En lo que respecta al deslizamiento de la similitud histórica a la disculpa moral, éste sería el producto de un proceso de comparación compensatoria de los crímenes entre sí: si los turcos llevaron a cabo un genocidio con los armenios y Stalin instauró el régimen de Gulags, por lo tanto, habría una suerte de contexto justificatorio y disculpatorio de los campos de concentración y exterminio nacionalsocialistas. Sin embargo, semejante comparación resulta falaz: “...el error sería confundir la excepcionalidad absoluta en el plano moral con la incomparabilidad relativa en el plano historiográfico." (Ricoeur 2000, p. 435). A nivel moral, no hay una escala de lo inhumano, lo inhumano está fuera de toda escala.

Haber rechazado todas estas estrategias de articular el plano histórico y el moral, no significa que Ricoeur rechace de plano que sea posible. Como adelantamos al comienzo de este artículo, consideramos que la clave para comprender su propuesta se encuentra en los artículos que hiciera en torno al testimonio. Nos retrotraeremos, por lo tanto, al artículo "Emmanuel Levinas, penseur du témoignage" de fines de los ochenta para luego establecer su influencia en La memoria, la historia, el olvido.

\section{III}

En el artículo en cuestión se lleva a cabo una lectura comparativa del tema del testimonio y la atestación en Levinas, Nabert y Heidegger, utilizando como eje de lectura la correlación entre Altura y Exterioridad. El eje la Altura, es decir, la asimetría entre quien testifica y quien recibe el testimonio, permite comparar a la conciencia heideggeriana, la afirmación originaria de Nabert y la 'gloria del infinito' de Levinas. Por su parte, el eje de la Exterioridad, esto es, la distancia que separa a ambos polos del testimonio, reúne la no familiaridad heideggeriana, la mediación de otras conciencias que testifican absolutamente lo absoluto en Nabert y la condición levinasiana de rehén 5 . La tesis de este artículo es que “...Altura y/o Exterioridad no constituyen solamente por su unión los aspectos destacables de los filósofos del testimonio, sino que proporcionan además el criterio de su diferencia, en la medida en que, como voy a mostrar, de Heidegger a Nabert, de Nabert a

\footnotetext{
5 Cf. Greisch (1995), p. 308.
} 
Levinas, el gradiente de la superioridad crece con el de la exterioridad"6. Dos motivos nos llevan a retrotraernos a este artículo. En primer lugar, en él se lleva a cabo una caracterización filosófica del testimonio y la atestación de los tres filósofos más influyentes en la ontología de la condición histórica ricoeuriana, despojada de todo componente fáctico. Consideramos que estas caracterizaciones siguen presentes en La memoria, la historia, el olvido, con el inconveniente de que se desdibujan detrás de debates históricos. Este rodeo, entonces, nos facilitará la identificación de las distintas posiciones filosóficas subyacentes en la obra que nos convoca. El segundo motivo apunta a reforzar la hipótesis de la cercanía entre Ricoeur y Nabert. Si este artículo, signado por la problemática de la identidad personal y dedicado a Levinas, concluye señalando la importancia de complementar el pensamiento de Levinas con el de Nabert, es de esperar que una obra dedicada al problema de la representación mnémica e histórica la afinidad con Nabert sea aún mayor.

Heidegger toma distancia respecto de las filosofías de la reflexión al incorporar al testimonio en calidad de dimensión dóxica no reductible a la meramente epistémica. Mientras para éstas hay una coincidencia entre el sí que se conoce y el conocido, la analítica existenciaria recurre al testimonio para asegurarse la originariariedad de la caracterización ontológica del Dasein como cuidado. Según Ricoeur, la estrategia de Heidegger habría consistido en beneficiarse de la fuerza superior de la autentificación de la conciencia, sin concederle a ésta ninguna trascendencia. Para que esto fuera posible, fue preciso llevar a cabo una doble reducción de la trascendencia. En el primero se reduce el contenido. Lo atestado es simplemente el poder ser sí mismo en su carácter existencial y existenciario, careciendo de cualquier criterio de distinción entre lo bueno y lo malo. Esta reducción del contenido de lo atestado impide la apertura de la analítica existenciaria a la dimensión ética. La segunda reducción cierra el acceso al otro. Quien voca en el testimonio es el Dasein mismo, el cual, habiendo precursado su propia muerte, voca al sí impropio desde su propiedad. Esta reducción acaba siendo más radical que la anterior puesto que, como veremos más adelante, la apertura al otro es la condición de posibilidad de la remisión a la dimensión ética. Por lo tanto, aún cuando lo atestado careciera de todo contenido moral, la apertura a los semejantes acabaría vinculándonos con esta dimensión. Esta doble reducción llevada a cabo por Heidegger acaba superponiendo a la Altura y la Exterioridad en la figura del Dasein: "En este punto la interpretación del tema de la altura se superpone con el de la exterioridad. A una altura sin trascendencia corresponde una exterioridad sin alteridad." (Ricoeur 1989, p. 19). De este modo, la falta de una dimensión ética y la ausencia del otro llevan a que su concepción del testimonio sea la de menor grado de Altura y Exterioridad de los tres autores tratados.

6 Cf. Ricoeur (1989), p. 18. 
Ricoeur presenta a Nabert como aquel filósofo que, a diferencia de Heidegger, introduce el corte ético en la ontología y que permite moderar algunas de las tesis hiperbólicas de Levinas. En este artículo, Nabert es introducido de una manera similar a como lo había sido en "La Hermenéutica del Testimonio", es decir, como un pensamiento que intenta articular la "ruptura ética" que se produce a partir de lo injustificable, por una parte, y la correlación entre la criteriología de lo divino y la hermenéutica del testimonio, por la otra. Según Ricoeur, en Elementos para una ética Nabert abre la dimensión ética a partir de las experiencias de pasividad recogidas por los sentimientos y no por medio de la razón. Esto significa que, a diferencia de la filosofía kantiana, existen múltiples fuentes de reflexión ética. De todas estas pasividades, la protesta contra lo injustificable es la que nos hace reconocer la desmesurada Altura de la conciencia absoluta. Lo injustificable es definido como aquello que no se puede medir meramente por la violación de las normas morales, y por lo tanto, no puede ser explicado desde la buena voluntad. Ante esta incapacidad de nuestros recursos para comprenderlo, acabamos recurriendo al testimonio de otros que sí lo han vivido. En este punto, se establece el vínculo entre la Altura y la Exterioridad a través de la atestación. En efecto, Nabert es consciente de que no hay certeza de lo que se testifica y que la confianza que se le concede al testigo está constantemente rodeada por un halo de sospecha.

A diferencia de lo que sucede en Levinas y Heidegger, la Altura y la Exterioridad no coinciden en la misma persona. Los testimonios dan cuenta de acontecimientos reales que ninguna reflexión podría sacar de sí. Se produce, entonces, un desdoblamiento del testimonio al exterior de la reflexión. Habría un testimonio dado por alguien que no ha deseado vivir esa experiencia, pero espera que su testimonio sea comprendido. Habría, por otra parte, un testimonio-narración de segundo grado realizado a partir de los testimonios anteriores por los testigos de los testigos. Entre ambos tipos de testimonios se establece una estructura dialógica: "alguien dona un signo de lo absoluto, sin quererlo ni saberlo; otro lo interpreta como signo." (Ricoeur 1989, p. 27).

Por último, se caracteriza a Levinas como la contracara de Heidegger, en el sentido en que toma al otro como la figura central de su pensamiento y supedita toda consideración filosófica a la ética. Según este autor, el uno-para-el-otro, constitutivo de mi responsabilidad, es del orden de la asignación y no de la manifestación. Abordar ontológicamente al otro conduce a su ocultamiento, ya que el interés de la ontología es la representación y la manifestación. Incluso las filosofías de la conciencia son ontologías, cuyo objetivo consiste en proporcionar respuestas al problema del principio, de la arche. De lo que se trata, entonces, es de abordar al otro, sin por ello desnaturalizarlo. Para lograrlo, Levinas evita, en primer lugar, tanto el uso del lenguaje representativo como así también el tradicional interés por la arche. En lugar de ello, se retrotrae a un ámbito previo a todo comienzo, a una an-archia. Esta 
an-archia también es definida como un pasado más viejo que todo pasado rememorable, una pasividad que no es la inversa de la actividad, una libertad diferente a la de la iniciativa o una acusación previa a la falta. Según Ricoeur, “... es por esta primera estrategia, por la que se conquista la Altura de aquello que nos ha precedido desde siempre. En varias ocasiones, Emmanuel Levinas evoca la creación y la condición de criatura como aquello que no puede ni debe ser pensado de manera ontológica." (Ricoeur 1989, pp. 32-33). La segunda estrategia para evitar desnaturalizar al otro consiste en recurrir a expresiones hiperbólicas a fin de desconcertar al pensamiento común y reforzar la Exterioridad del otro que me asigna a la responsabilidad. Aún cuando es posible afirmar que el testimonio ocupa un lugar marginal en el pensamiento de este filósofo, en opinión de Ricoeur, cumple un papel central dentro de esta doble estrategia. El testimonio es un modo de verdad opuesto al de la certeza de la representación que permite reunir la Altura y la Exterioridad. "No se trata finalmente de otro testimonio dado a la Altura, a la gloria de lo infinito que el testimonio de la Exterioridad, de la asignación a la responsabilidad." (Ricoeur 1989, p. 36).

Habiendo presentado las posiciones de estos tres filósofos, Ricoeur se propone darle una vuelta de tuerca al pensamiento de Levinas y mostrar el modo en que éste nos conduce a la problemática de la ipseidad. El distanciamiento respecto de las filosofías de la conciencia lleva al abandono de la noción de conciencia y de toda concepción de identidad como mismidad. Sin embargo, la responsabilidad requiere un quién, un sí mismo, no tematizable sino gobernado por la gramática del acusativo. Como bien señala Greisch, "Ricoeur muestra que el pathos levinasiano del 'desdecir', si le da completamente la espalda a la identidad-mismidad, no hace otra cosa que poner paradojalmente a la luz la identidad-ipseidad del sí en su condición de rehén." (Greish 1995, p. 309). Con todo, Ricoeur es consciente que estas consideraciones, cercanas a su ontología del hombre capaz, son incompatibles con la absoluta pasividad que caracterizan al sujeto levinasiano. Es por ello por lo que acaba reconociendo; "El sí, ¿sería resultado, si no fuera en primer lugar presuposición, es decir, potencialmente capaz de entender la asignación? Lo sé bien, indagar sobre alguna capacidad, sobre alguna potencialidad que no fuera la obra misma de la asignación, es para Levinas plantear una cuestión inadmisible." (Ricoeur 1989, p. 38). Será esta incompatibilidad la que lo obliga a concluir el artículo reuniendo a Levinas con el pensamiento de Nabert.

Acabamos de presentar la visión ricoeuriana de finales de la década del ochenta sobre el papel del testimonio en Levinas, Heidegger y Nabert. A continuación mostraremos que sus aspectos salientes continuarán presentes en La memoria, la historia, el olvido, aunque el desplazamiento del interés de la identidad personal hacia la historia agudizará la afinidad con Nabert. Este paralelismo nos proporcionará el sustento necesario para recurrir a las consideraciones que Ricoeur había rea- 
lizado sobre el testimonio absoluto de lo absoluto en Nabert para aplicarlo a los testigos del Holocausto, aún cuando no haya referencias explícitas al respecto.

\section{IV}

La ontología heideggeriana es uno de los pilares teóricos de La memoria, la historia, el olvido. La temporalidad es señalada como la condición de posibilidad de la epistemología de la historia7, y el existenciario de la deuda, el fundamento ontológico de la representancia ${ }^{8}$. Sin embargo, Ricoeur mantiene su crítica a la ausencia del componente moral de la deuda y el solipsismo en el que cae el Dasein. A diferencia de lo que había sucedido diez años antes, en esta obra propone una alternativa para salvar estas limitaciones. De este modo, nos encontramos con una cita como la siguiente: "pienso que la idea de falta debe retomar su lugar a un estadio bien preciso del juicio histórico, desde que la comprensión histórica se confronta con agravios realizados; la noción del agravio hecho al otro preserva, pues, la dimensión propiamente ética de la deuda, su dimensión culpable." (Ricoeur 2000, p. 473).En tanto el vínculo con el otro es la condición de la apertura de la deuda a la dimensión ética, su modificación supondría la posibilidad de acceder a esta dimensión. Para lograrlo, se vuelve preciso repensar a la muerte como la posibilidad más propia. Antes de considerar al ser humano como un ser para la muerte, Ricoeur se encuentra más inclinado a posiciones como las de Levinas o Sartre, que la toman como una suerte de corte impuesto a la vida. "E. Levinas es claro y firme sobre el frente-a-la-muerte, que no puede ser sino un contra-la-muerte y no para-lamuerte." (Ricoeur 2000, p. 470). Esta concepción de la muerte permite incorporar el componente violento que supone la interrupción de una vida y la protesta que uno debe levantar contra ella.

Con el pretexto de que el precursar-la-muerte heideggeriano imposibilita la articulación de la analítica existenciaria con la historia, constantemente vinculada con la muerte de los demás, Ricoeur plantea la necesidad de incorporar al otro en el proceso de la toma de conciencia de la muerte. Con el objeto de restituir la densidad ontológica del "se muere" impropio", se propone reemplazar la denominada vía corta heideggeriana, que establece un vínculo directo entre el poder-ser y la muerte, por otra larga, que abra al Dasein a la exterioridad y la factualidad. La exteriori-

${ }^{7}$ Cf. Ricoeur (2000), p. 454: “...la temporalidad constituye la precondición existencia de la referencia de la memoria y de la historia al pasado."

8 Cf. Ricoeur (2000), p. 473: "Este concepto de deuda-herencia viene a ubicarse debajo del de la representancia propuesto en el cuadro de la epistemología del conocimiento histórico como guardián de la pretensión referencial del discurso histórico [...] El ser-en-deuda constituye en este sentido la posibilidad existencial de la representancia."

${ }^{9}$ Cf. Ricoeur (2000), p. 475. 
dad se logra introduciendo al cuerpo propio y a la carne en la analítica existenciaria. Esta incorporación permite reconocer que, desde el punto de vista genético, nuestro primer contacto con la muerte surge de la observación de cómo la muerte se presenta en la naturaleza como un obstáculo al deseo de vivir. La factualidad, por su parte, nos remite al contacto con la muerte a través de mis semejantes. El abismo que separa estas primeras experiencias fácticas respecto de la posición existenciaria de Heidegger, en la que el precursar-la-muerte es parte de la comprensión del sí, se cierra en un proceso de introyección.

En lo que a Levinas respecta, Ricoeur coincide en su planteo de "Verdad como manifestación y verdad como testimonio" en que "es en el orden de la verdad donde el testimonio constituye una noción alternativa con respecto tanto al orden de las filosofías ontológicas cuanto a las filosofías de la conciencia." (Ricoeur 1989, p. 34).Con otras palabras, el testimonio pone de manifiesto un modo de verdad alternativo al de la certeza de la ontología y que está presente en toda la dimensión ética. Sin embargo, el carácter antirepresentativo del testimonio levinasiano no se condice con los hechos ni con el pensamiento de Ricoeur, para quién el testimonio se caracteriza por ser una representación atestable de un acontecimiento pasado. En $L a$ memoria, la historia, el olvido el testimonio es caracterizado por seis componentes esenciales: 1. la articulación de la aserción de una realidad fáctica de un acontecimiento pasado, junto con la certificación de la declaración por la experiencia de su autor; 2. la inseparabilidad de la aserción fáctica con la auto-designación del sujeto que atestigua; 3 . La inscripción del testimonio en una situación dialógica; 4. La controversia que abre la sospecha: 5. La capacidad de mantener la palabra; 6. La estabilidad de la disposición a testificar como factor de seguridad en el conjunto de las relaciones constitutivas del vínculo social ${ }^{10}$. El primero de estos atributos pone de manifiesto la imbricación de las dos dimensiones que Levinas considera incompatibles: la de ser una representación de un acontecimiento pasado y la de ser la atestación de quien lo ha vivido.

Por una parte, es una representación del pasado. Al comienzo de la obra que nos convoca nos encontramos con la siguiente afirmación: "una problemática común corre en efecto a través de la fenomenología de la memoria, la epistemología de la historia, la hermenéutica de la condición histórica: aquella de la representación del pasado. [...] La misma pregunta atraviesa la epistemología del testimonio, luego aquella de las representaciones sociales tomadas por objeto privilegiado de la explicación/comprensión, para desplegarse en el plano de la representación escritural de los acontecimientos, coyunturas y estructuras que puntúa el pasado histórico." (Ricoeur 2000, p. II). Allí queda manifiesto que el testimonio es una representación. Sin embargo, por la otra, este tipo de representación no puede aspirar a la verdad

10 Cf. Ricoeur (2000), pp. 204-206. 
propia de una representación histórica, sino a la fidelidad que caracteriza a la atestación. Es esta peculiaridad del testimonio la que obliga a que el filósofo francés tome distancia del que denomina "paradigma de registro" de Dulong, pues reduce el testimonio a un lenguaje corroborable.

En lo que respecta a Nabert, Ricoeur caracteriza la encrucijada en la que el Holocausto coloca a la historia de una manera análoga a la que se encuentra al comienzo de su filosofía de lo absoluto. Hemos señalado que esta reflexión se apoya en dos ejes: la protesta contra lo injustificable que obliga a la conciencia empírica a reflexionar sobre lo absoluto 11 y la crisis del testimonio, que impide que la conciencia tenga certeza absoluta sobre lo testificado ${ }^{12}$. Estos mismos componentes se encuentran presentes en La memoria, la historia, el olvido. Por una parte, hemos recogido en el debate sobre la singularidad histórica del Holocausto la cita en la que Ricoeur recurre a la expresión nabertiana de injustificable para caracterizar dicho acontecimiento histórico13. En lo que respecta a la crisis del testimonio, es imposible desconocer los efectos del Holocausto en su uso histórico. En la modernidad, el testimonio fue tomado como uno de los ejes de la separación de la historia sacra y la laica. Autores como Locke y Hume propusieron la conformidad con el conocimiento, la observación y la experiencia común como criterio de aceptabilidad de un testimonio ${ }^{14}$. Este criterio obligaba a que el historiador descartara a los testimonios acerca de los milagros como evidencia histórica. Sin embargo, los horrores padecidos por los sobrevivientes del Holocausto exceden la comprensión y la experiencia media de la población. De haberse continuado con este criterio, se deberían haber excluido estas declaraciones a la hora de escribir su historia, perdiéndose la fuente de evidencia histórica más rica sobre este acontecimiento. "Se trataba de luchar contra la credulidad y la impostura; se trata ahora de luchar contra la incredulidad y la voluntad de olvidar." (Ricoeur 2000, p. 223).Ambos ejes, protesta frente a lo injustificable y crisis del testimonio, son reunidos en el corolario que hace Ricoeur al reclamo de Carlo Ginzburg para que se reconozca el testimonio de un solo sobreviviente. "La súplica a favor de la realidad del pasado histórico, similar a aquel de Vidal-Naquet en Los judios, la memoria, el presente y en Los asesinos de la memoria [...] reviste así el doble aspecto de una atestación incontestable y de una protesta ética que prolonga la violencia del impulso que mueve a un sobreviviente como Primo Levi a testimoniar. Es sobre este entrelaza-

11 Cf. Ricoeur (1989), p. 24: "pero es en la protesta contra lo injustificable, sobre la cual se abre el Ensayo sobre el mal, que la consciencia empírica toma la medida desmesurada de la Altura de la consciencia absoluta."

12 Cf. Ricoeur (1972), p. 55: "La hermenéutica nace ahí, una segunda vez; no hay manifestación de lo absoluto sin la crisis del falso testimonio, sin la decisión que existe entre el signo y el ídolo."

13 Cf. Nota 8 de este artículo.

14 Cf. Coady (1992) p. 82. 
miento de la atestación y de la protesta en el caso de la literatura de la Shoah sobre lo que hace falta reflexionar." (Ricoeur 2000, p. 334).

Teniendo en cuenta estos paralelismos explícitos entre el modo en que Nabert inicia su filosofía de lo absoluto y la caracterización ricoeuriana del Holocausto, quisiera volver a la problemática de la singularidad, y transponer algunos de los componentes de la hermenéutica del testimonio nabertiana con el objeto de esclarecer su papel dentro de la articulación entre la historia y la ética.

\section{V}

Tras haber criticado las dos alternativas que se presentaban para articular la singularidad histórica y la moral, Ricoeur propone otra alternativa. "¿No existe, pues, ningún vínculo asignable entre el uso moral de las ideas de unicidad y de comparabilidad y su uso historiográfico? Yo percibo uno, que sería la idea de ejemplaridad del singular. Ésta no depende ni de la estimación moral en tanto que tal, ni de la categorización historiográfica, ni de su superposición, que sería un retorno a lo equívoco, a la confusión." (Ricoeur 2000, p. 435).

¿En qué consistiría esta articulación y cuál es el papel del testimonio? Para entender este punto, debemos aplicar algunas de las categorías de Nabert arriba recogidas a la investigación de la historia. No hay lugar a dudas de que el testigo de lo injustificable es el sobreviviente del Holocausto, que proporciona el signo de lo absoluto, de lo moral, sin saberlo ni haberlo querido. Lo que puede llevar al debate es quién es el intérprete de este signo: éste no es el historiador, sino el ciudadano. Él es, en definitiva, quien atesta el testimonio y protesta contra lo injustificable, buscando que no se repitan. Así explica Ricoeur, "la idea de singularidad ejemplar sólo puede formarse por una opinión pública ilustrada que transforma el juicio retrospectivo referido al crimen en juramento para evitar su retorno." (Ricoeur 2000, p. 435). Cuando se habla de ejemplaridad no se está pensando en un caso que ilustra la ley, situación que Nabert rechaza como marco de su filosofía de lo absoluto a favor del testimonio de lo injustificable. Este concepto está antes bien ligado a la distinción de Todorov entre lo literal y lo ejemplar ${ }^{15}$. Justamente en su mención de lo ejemplar nos describe el modo en que transforma el dolor de pasado hacia un futuro mejor, tal como se señala en la última cita, transformación en la cual la labor crítica e ilustradora del historiador cumple un papel de relevancia. "Esta preocupación enlaza con los objetivos precedentes aprovechando el consejo realmente atinado de Todorov de extraer de los recuerdos traumatizantes el valor ejemplar que sólo el trastrocamiento de la memoria en proyecto puede hacer pertinente. Si el traumatismo remite al pasado, el valor ejemplar orienta hacia el futuro." (Ricoeur 2000, p.

15 Cf. Todorov (2000), p. 30. 
105). Queda, pues, aclarar cómo se forma la idea de singularidad ejemplar, y el modo en que el historiador ilustra al ciudadano, para lo cual deberemos retrotraernos a las consideraciones más epistemológicas acerca del testimonio.

Una vez caracterizado el testimonio por medio de sus seis atributos arriba resumidos, Ricoeur establece el modo en que éste se convierte en parte de la base documental del historiador. A grandes rasgos, esta conversión es sólo posible a partir de la labor de trascripción, preservación y clasificación de las declaraciones orales por parte del archivista. La transcripción introduce el elemento crítico que asegura la objetividad de lo declarado en el testimonio. “...Estos testimonios orales sólo constituyen documentos una vez registrados; dejan la esfera oral para entrar en la de la escritura, se alejan así de la función del testimonio en la conversación ordinaria. Se puede afirmar entonces que la memoria está archivada, documentada. Su objeto ha dejado de ser un recuerdo en el sentido propio del término, es decir, retenido en relación de continuidad y de apropiación respecto a un presente de la conciencia." (Ricoeur 2000, p. 226).Al proceder según el ideal del paradigma de registro, el archivista preserva el carácter contrastable de las pruebas y, por lo tanto, la pretensión de verdad en historia ${ }^{16}$.

Hemos mencionado que el testimonio oral está vinculado con la memoria, lo que significa que esta declaración está directamente ligada al acontecimiento pasado, pero a la única ambición a la que puede aspirar es a la fidelidad, es decir, a mantenerse a lo largo del tiempo. Una vez que el archivista ha filtrado críticamente el testimonio y lo ha fijado de manera escrita, pierde ese vínculo directo con el pasado y con el testigo. Esto significa, por una parte, que deja de estar ligado con un acontecimiento histórico, para constituirse en un hecho histórico, y por la otra, que, en lugar de aspirar a la fidelidad, lo establecido por esta proposición pretende ser verdadero. "Un recuerdo archivado ha dejado de ser en el sentido propio de la palabra un recuerdo, es decir, algo que mantiene una relación de continuidad y de pertenencia con un presente del que se es consciente. Ha adquirido el estatuto de resto documental." (Ricoeur 1995, p. 45).

Si Ricoeur se hubiera limitado a reducir el testimonio a ser evidencia documental, hasta aquí hubiera llegado el alcance de las declaraciones de los sobrevivientes, y el ciudadano no hubiera podido tener acceso a ellas. Sin embargo, el filósofo nos advierte sobre este punto del siguiente modo: "en este capítulo seguiremos sólo uno de los destinos del testimonio, sellado por su puesta en archivo y sancionada por la prueba documental." (Ricoeur 2000, p. 202). El componente evidencial es sólo un aspecto del testimonio, y no puede quedar identificado con él. Menos aún si se trata de un testimonio de un acontecimiento injustificable. “...Estos testimonios directos se encuentran progresivamente encuadrados, pero no absorbidos por los trabajos de historiadores del tiempo presente y por la publicidad de los grandes procesos crimi-

16 Cf. Ricoeur (2000), p. 443. También Ricoeur (1996), p. 71. 
nales de los cuales las sentencias avanzan lentamente en la memoria colectiva a costa de penosos dissensus." (Ricoeur 2000, p. 224).El ciudadano tiene acceso directo al testimonio de los sobrevivientes como lo ejemplifica, entre otros, el éxito editorial que ha tenido la obra de Primo Levi, Elie Wiesel, Jorge Semprún o Víctor Frankl. Lo que aporta el historiador es la dimensión crítica cuyo distanciamiento impide que el ciudadano caiga en una memoria literal.

A lo largo del tiempo, el concepto de testimonio ha afectado el modo en que se concibió a la historia y a la labor del historiador. En la Ilustración, autores como Locke y Hume propusieron como criterio de aceptabilidad de un testimonio a la conformidad con el conocimiento, la observación y la experiencia común. Este criterio obligaba a que el historiador descartara como evidencia histórica los testimonios acerca de los milagros, y condujo a la separación entre historia sacra e historia laica. El Holocausto ha dado lugar a una nueva crisis del testimonio que obliga a repensar algunas de las conclusiones y criterios que habían surgido en la Ilustración. Los horrores padecidos por los sobrevivientes del Holocausto exceden la comprensión y la experiencia media de la población, por lo que, de haberse continuado con el criterio de aceptabilidad ilustrado, deberían haberse rechazado.

Volviendo a lo planteado al comienzo de este artículo, La memoria, la historia, el olvido puede ser visto como un abordaje epistemológico de la filosofía de la historia, en que el testimonio pueda ser definido simplemente "...la estructura fundamental de transición entre la memoria y la historia." (Ricoeur 2000, p. 26).Consideramos, empero, que a lo largo de estas páginas ha quedado de manifiesto que este libro pretende señalar el lugar que tiene la ética en la historia. El Holocausto ha dado lugar a una crisis del testimonio, sobre la que Ricoeur ha reflexionado. Quisimos mostrar que el testimonio no es meramente parte de la base documental de la historia, sino que tiene un papel mucho más complejo, que fue preciso desentrañar. Entre las consecuencias más importantes de esta lectura nos encontramos que las dos figuras principales dejan de ser la comunidad y el historiador y pasan a ser el sobreviviente del Holocausto, en su calidad de testigo de lo injustificable y el ciudadano, en tanto intérprete. Nos volvimos al artículo "Emmanuel Levinas, pensador del testimonio" por su análisis de los que consideramos son los tres pilares de la ontología de la condición histórica en Ricoeur: Heidegger, Nabert y Levinas. Este artículo nos proporcionó los elementos necesarios para discriminar detrás de los debates historiográficos recogidos, cuáles son los lineamientos teóricos de nuestro filósofo. Estos elementos, asimismo, nos proporcionaron el sustento necesario para transponer algunas de las afirmaciones que habí- 
an sido hechas sobre el testimonio de lo injustificable en Nabert al testimonio de los sobrevivientes del Holocausto, aún cuando no se lo sostuviera explícitamente.

Ricoeur no pretende volver a una posición acrítica en la que se acepte el testimonio sin más. Tampoco considera pertinente continuar con el ideal ilustrado de reducirlo simplemente a su base empírica. Una narración histórica que se limitara a reunir todos los hechos y estadísticas acerca del Holocausto dejaría de lado su aberración, que es en definitiva lo que lo define. El Holocausto, como hecho histórico, puede ser resumido en una larga serie de estadísticas, pero hay un excedente de sentido, ligado a cuestiones éticas, que se escapan de estos números. Su propuesta, en cambio, pretende recoger ambos aspectos del testimonio, el elemento fáctico y el de sentido. El primero será reunido por el historiador, luego de haber sido corroborado y asentado por el archivista. El segundo surge de la reflexión que lleva a cabo el ciudadano a partir del testimonio de los sobrevivientes de lo injustificable. La recepción no será empero acrítica, sino que, por el contrario, ésta se encontrará mediada por el tamiz del historiador. Esta reflexión no buscará quedarse atada al pasado, sino proyectarse hacia el futuro, para no repetir los errores de quienes nos precedieron.

\section{Referencias bibliográficas}

Alexander, J. C. (2004): "On the Social Construction of Moral Universals. The 'Holocaust' form War Crime to Trauma Drama" en ed. Alexander Jeffrey C., Cultural Trauma and Collective Identity, Berkeley - Los Angeles - London, University of California Press.

BEGUÉ, M.F. (2006): "El estatuto epistemológico del testimonio. Una manifestación diferente de la verdad" en Mena Malet, P. (comp.), Fenomenología por decir. Homenaje a Paul Ricoeur, Chile, Universidad Alberto Hurtado.

CoAdy, C. A. J. (1992): Testimony. A Philosophical Study, Clarendon Press, Oxford. Greisch, J. (1995): "Témoignage et attestation" en Greisch, J. (comp), Paul

Ricoeur. L’herméneutique à l'école de la phénoménologie, París, Intitut Catholique de Philosophie.

LeVI, P. (2006): Trilogía de Auschwitz, Barcelona, Océano.

LythGOe, E. (2011): "Paul Ricoeur y la representación histórica", Alea 9, pp. 75-94 Ricoeur, P. (1972): "L'Hermeneutique du Témoignage”, Archivio di Filosofia 42 $\mathrm{N}^{\mathrm{o}} 1-2$.

Ricoeur, P. (1989): "Emmanuel Lévinas, Penseur du Témoignage" en Aeschlimann, J.C. (ed.), Répondre d'autrui. Emmanuel Lévinas, BoudryNeuchâtel, Éditions de la Baconnière.

Ricoeur, P. (1996): Sí mismo como otro, México, Siglo XXI. 
Ricoeur, P. (1999): La lectura del tiempo pasado: memoria y olvido, Madrid, Universidad Autónoma de Madrid.

Ricoeur, P. (2000): La mémoire, l'histoire, l'oublie, Paris ,Seuil.

Todorov T. (2000): Los abusos de la memoria, Barcelona, Paidós.

Esteban Lythgoe

CONICET - Argentina

estebanlythgoe@yahoo.com.ar 